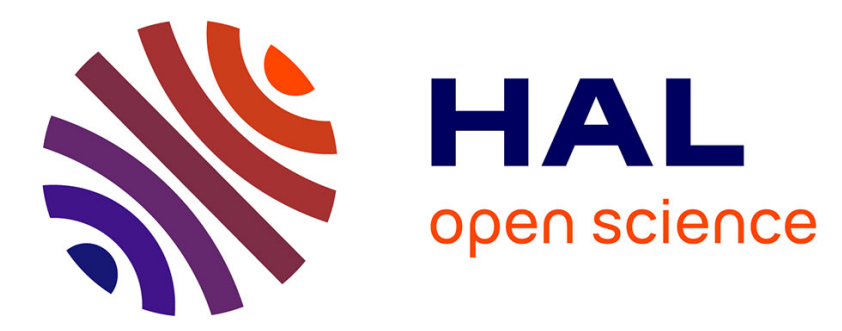

\title{
Visual perception driven registration of mammograms
}

Arnaud Boucher, Florence Cloppet, Nicole Vincent, Pierre Jouve

\section{To cite this version:}

Arnaud Boucher, Florence Cloppet, Nicole Vincent, Pierre Jouve. Visual perception driven registration of mammograms. 20th International Conference on Pattern Recognition (IPCR 2010), Aug 2010, Istanbul, Turkey. 10.1109/ICPR.2010.581 . hal-00643193

\section{HAL Id: hal-00643193 \\ https://hal.science/hal-00643193}

Submitted on 24 Nov 2011

HAL is a multi-disciplinary open access archive for the deposit and dissemination of scientific research documents, whether they are published or not. The documents may come from teaching and research institutions in France or abroad, or from public or private research centers.
L'archive ouverte pluridisciplinaire HAL, est destinée au dépôt et à la diffusion de documents scientifiques de niveau recherche, publiés ou non, émanant des établissements d'enseignement et de recherche français ou étrangers, des laboratoires publics ou privés. 


\section{Visual perception driven registration of mammograms}

\author{
A. Boucher, F. Cloppet, N. Vincent \\ Paris Descartes University - Lipade (SIP) \\ Paris (FRANCE) \\ \{ arnaud.boucher ; florence.cloppet ; \\ nicole.vincent $\} @$ mi.parisdescartes.fr
}

\author{
P. Jouve \\ Fenics Society \\ Lyon (FRANCE) \\ pjouve@fenics.fr
}

\begin{abstract}
This paper aims to develop a methodology to register pairs of temporal mammograms. Control points based on anatomical features are detected in an automated way. Thereby, image semantic is used to extract landmarks based on these control points. A referential is generated from these control points based on this referential the studied images are realigned using different levels of observation leading to both rigid and non-rigid transforms according to expert mammogram reading.
\end{abstract}

Keywords - registration; control points; mammogram; anatomical features

\section{INTRODUCTION}

Breast cancer is the most prevalent cancer among women [1]. The majority of new breast cancers are diagnosed as a result of an abnormality seen on a mammogram, it is at the moment the best way to detect breast cancers in early stages. This has yielded to a resultant improvement in survival rates. Mammograms are X-ray images, where 3D-objects such as pectoral muscle, nipple, and adipose tissue, are projected in 2D dimensions (see Fig. $1)$.

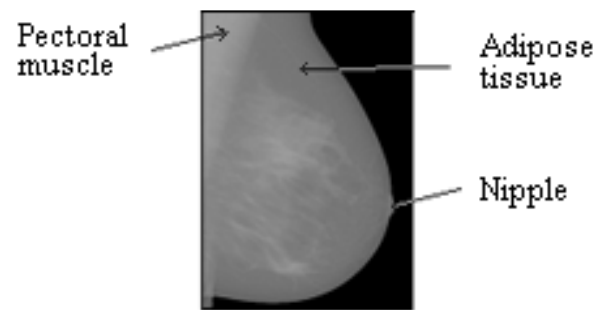

Figure 1 - Example of a MLO view mammogram

Several mammograms are done during a woman life. Such temporal information is used by radiologists. To provide an efficient solution to the problem of comparison of digital mammograms, a registration step is necessary. It is useful to reduce differences due solely to positioning the breast with respect to the device while preserving differences in mammograms caused by breast changes due to the apparition or progression of a disease. This is an important issue before a computed-aided diagnosis can be performed.

The purpose of this paper is to develop an automated method to register a couple of temporal mammograms in mediolateral-oblique (MLO) view. The method is inspired by experts reading methods and based on the patient anatomy. After presenting the problem of mammogram registration in section 2 , our contribution will be detailed in section 3 . The results are discussed in section 4.

\section{MAMMOGRAM REGISTRATION}

The mapping of information given by several multi or mono modal images has been an active research topic in the biomedical field. If no registration is applied, it is difficult to localize and compare regions of interest on two images, as differences of size, orientation or other distortions may have occurred. Brown [2] defines the transform associated with a registration as follows:

$$
\mathrm{t}_{0}=\operatorname{argmax}_{\mathrm{t} \in \mathrm{T}} \mathrm{S}(\mathrm{I}, \mathrm{t}(\mathrm{J}))
$$

where $I$ and $t(J)$ are the referent and transformed images, compared using a similarity measure $\mathrm{S}$.

It consists in an estimation of an optimal transform $t$ belonging to $T$, which allows the mapping of two images $I$ and $J$; and argmax represents a resolution strategy. As illustrated by Figure 2, a transform $t$ can be rigid (translation, rotation), or non rigid (affine : global linear transformation; deformation field). On the one hand, rigid and affine transforms are widely used to recover global deformations, e.g. for intra-patient registration. However, they do not cope with local deformations. On the other hand, non-rigid transforms address the problem of local deformations; however they match contours of structure with local irregularities due to the high number of degrees of freedom.

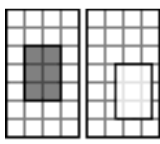

translation

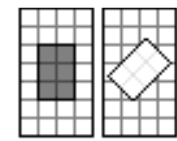

rotation

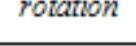

rigid
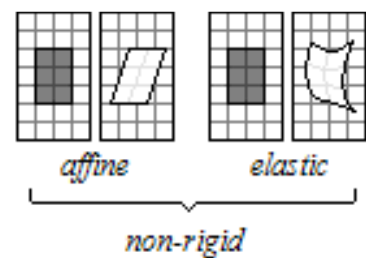

Figure 2 - Types of transformations

Various methods have been developed to correlate areas in mammograms in order to detect differences that are likely to be associated with an abnormality. A survey of these methods can be found in [1]. 
Most of the rigid methods rely on extraction of corresponding landmarks (points, curves or surfaces). These landmarks quality plays an important role in the alignment accuracy. Tedious is manual landmarks generation and it is a time-consuming task, therefore a large number of methods are based on an automated extraction of landmarks in mammograms. According to [3], the only robust corresponding landmarks for mammogram registration are anatomical features such as breast boundary, pectoral muscle, nipple, crossing points of horizontal and vertical structures, fibrous tissue, ducts and vessels, the latest are less robust in case of controlateral mammograms registration, due to the natural dissymmetry between left and right breasts. A lot of methods have been developed to extract robust anatomical features $[4,5,6]$. These robust anatomical features are not homogeneously spatially distributed. Therefore a rigid registration of mammograms needs an intelligent use of these anatomical features.

Most of non-rigid registration methods are iterative and minimize a cost or an energy function defined in terms of the intensity difference between images. The similarity criterion may involve mutual information (2) that implies entropy computation (3):

$$
\begin{aligned}
& \mathrm{IM}(\mathrm{I}, \mathrm{J})=\mathrm{H}(\mathrm{I})+\mathrm{H}(\mathrm{J})-\mathrm{H}(\mathrm{I}, \mathrm{J}) \\
& \mathrm{H}\left(\mathrm{I}_{\mathrm{k}}\right)=-\sum_{\mathrm{C}} \mathrm{p}_{\mathrm{i}} \cdot \log \left(\mathrm{p}_{\mathrm{i}}\right)
\end{aligned}
$$

where $I$ and $J$ are the processed images, $C$ is the set of $\mathrm{n}$ gray levels, and $i$ is comprised between 1 and $\mathrm{n}$.

Other similarity measures can be used such as the sum of absolute differences, the sum of squared differences, a correlation coefficient or the Wood's criteria [7]. As these methods imply an iterative minimization of an energy function, they are quite time consuming. To decrease the iteration number, some methods use a rigid approach to initialise the search in a non-rigid method [8]. Moreover, some biomechanical models $[9,10]$ have been proposed to improve non-rigid registration, with adaptation to breast compression that implies deformation of the soft tissue. Another drawback of non-rigid methods is the potential modification of abnormalities evolving over time.

In the image mammograms registration, very important is to preserve differences caused by breast changes or new disease appearance or progress of existing disease. To preserve as much as possible these mammograms differences not caused by positioning, we propose a fully automated coarse-to-fine registration method. It is based on robust anatomical features linked to intensity and on semantical interpretation. We have been inspired by expert's perception and reasoning way.

Then local non-rigid transforms are possible. The joint use of a global rigid registration followed by a local affine method will allow us to simulate a deformation field while maintaining the processing speed of rigid methods.

\section{VISUAL PERCEPTION DRIVEN METHOD}

Most of the registration methods look for matching images either at a global level or at a local level according to a function to be optimized. Of course this is not consistent with expert reasoning and human vision processing. Different observation levels have to be taken into account. A global view focuses on anatomical elements that can easily be matched and that give sense to the different parts of the breast. Indeed, texture must not be used at this level as it is too much involved in the defaults that are the core of the mammogram reading. In a second step, some semantical meaning is associated with each region, with possibly more local processes. A raw matching is then done based on a rigid anatomic referential (a primary model) associated with each breast.

To simulate the estimation of a deformation field some independent affine transforms are applied to regions linked to this referential, as the experts practice. This approach allows simulation of elastic deformation and maintains a low computation time.

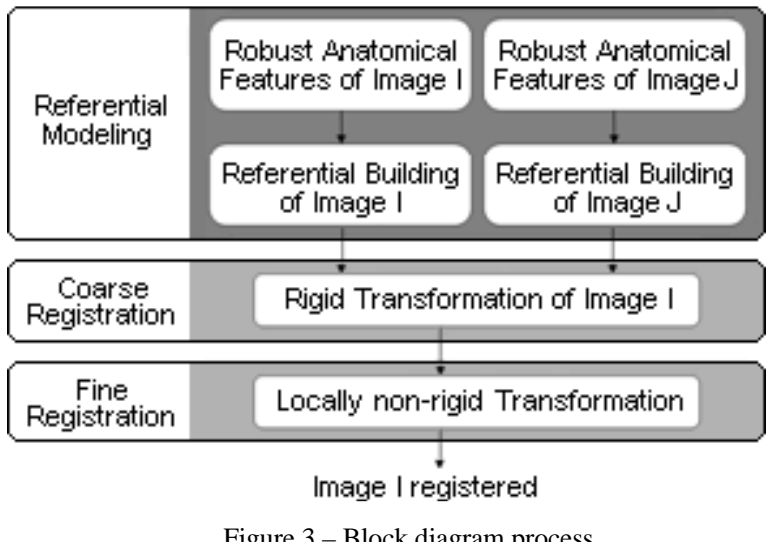

First, the anatomical elements enable the building of a referential associated with each breast image. The breast boundary, the pectoral muscle, the nipple, the lower (fold between the belly and the breast) and higher limits of the breast (see Fig.4) can be extracted in a robust way. They are referential points commonly used by experts.

As the paper aim is not anatomical features extraction, we only here describe briefly this step.

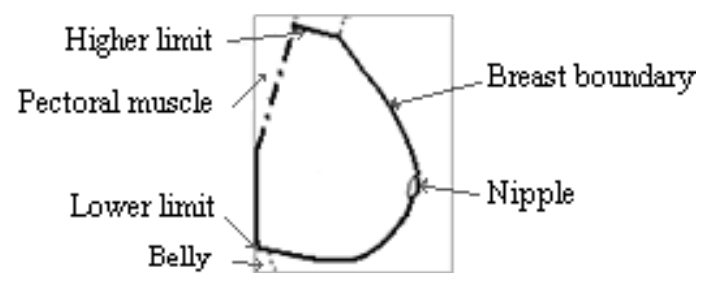

Figure 4 - Anatomical features extracted

Thresholding enables the breast boundary extraction. The nipple detection is based on a patch learning process. 
Random forests are used to generate decision trees that determine which region contains the nipple. A line approximating the pectoral muscle is obtained by use of the Hough transform.

This line is used as the $\mathrm{Y}$ axis of the referential to be built. The line perpendicular to $\mathrm{Y}$ axis and going through the nipple centre of gravity is chosen as $\mathrm{X}$ axis (see Fig. 5). The lower and higher limits of the breast are localized under and above the nipple axis where the breast thickness (distance from boundary breast to $\mathrm{Y}$ axis) is the smallest.

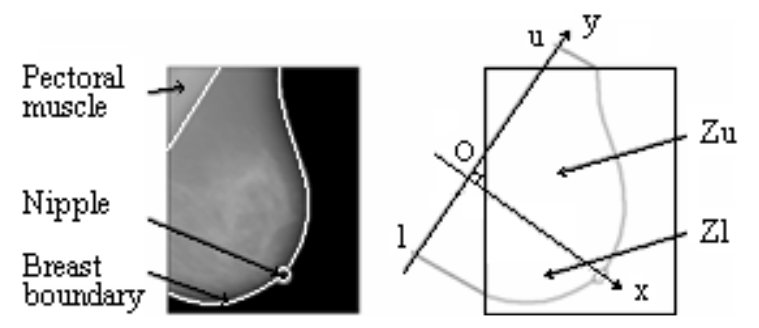

Figure 5 - Orthogonal system used as referential

The referential highlights two parts in the breast, the upper $Z_{u}$ and the lower part $Z_{1}$. Whereas they have the same texture, they are not deforming in the same way according to the acquisition process.

The registration is based on a patient referential and a two-step matching process. The first matching, scale independent, is a rigid transform. A translation, matches the origins of the referentials and a central rotation superimposes the axis. Let $\mathrm{F}$ be this transform. In the second step, only local processes are considered. The regions are inspired by the way the experts are reading the images. The experts vertically scan the image, therefore the $\mathrm{Y}$ axis is promoted. Then the scanning is modeled by way of horizontal rectangular zones $\left(\mathrm{Z}_{\mathrm{i}}\right)$, more precisely the zones are one pixel high. The images I and $\mathbf{J}$ are defined as:

$$
\mathrm{I}=\mathrm{U}_{\mathrm{l} \in \mathrm{LI}} \mathrm{Z}_{\mathrm{lI}} \quad \mathrm{J}=\mathrm{U}_{\mathrm{l} \in \mathrm{LJ}} \mathrm{Z}_{\mathrm{lJ}}
$$

where $l$ is the index of the zone $Z$ along the $\mathrm{Y}$ axis.

As noticed above, the stretching along the $\mathrm{Y}$ axis is not the same in upper part $Z_{\mathrm{u}}$ and in lower part $Z_{\mathrm{l}}$. Note these two pressures $\mathrm{V}_{\mathrm{Zu}}$ and $\mathrm{V}_{\mathrm{Zl}} \cdot \mathrm{V}_{\mathrm{a}}\left(\mathrm{Z}_{\mathrm{II}}\right)$ will be registered on $\mathrm{Z}_{\mathrm{IJ}}$ with $a$ taking $\mathrm{Z}_{\mathrm{u}}$ or $\mathrm{Z}_{\mathrm{l}}$ value.

The transformation between two segments with equal $y$ coordinates is modeled by a linear scaling, in the $\mathrm{X}$ axis direction. The scale changes are not identical all along the breast, figuring the different pressure the breast receives. We note $H_{b}$ the scale change applied on the horizontal with $b$ ordinate.

Let $S$ be a horizontal segment in image I :

$$
\mathrm{S}^{\prime}=\mathrm{V}_{\mathrm{a}}\left[\mathrm{H}_{\mathrm{b}}(\mathrm{S})\right]
$$

where $a$ designates $Z_{u}$ or $Z_{l}$ and $b$ is the $y$ coordinate of $S$ in the referential of image $\mathrm{J}$.
The breast referential allows to reduce differences due solely to positioning (orientation) of the breast during the acquisition step (see Fig.6), this is the coarse registration step. Then, during the fine registration step, the deformations are locally defined.
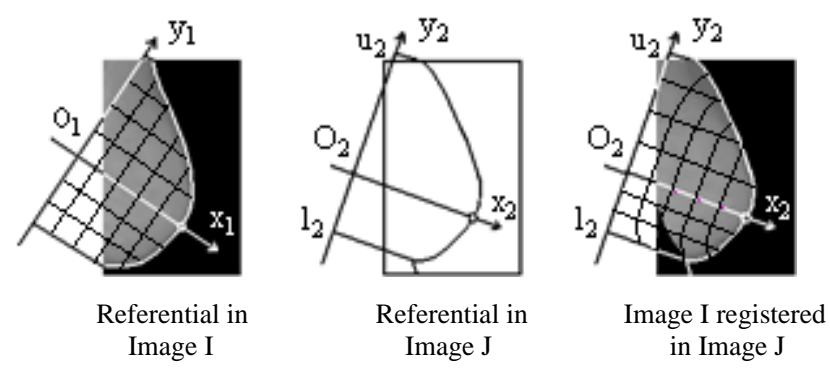

Figure 6 - Registration process of image I in image J

\section{RESULTS}

Evaluating registration algorithms is difficult due to the lack of gold standard in most clinical procedures [11]. The bronze standard is a real-data based statistical method that provides an alternative registration reference through a computationally intensive image database registration procedure. It is a statistical procedure whose purpose is to estimate the registration algorithms performances by considering the exact result as a hidden variable estimated from a large enough data set. The developed method was tested on 30 couples of temporal mammograms in MLO view. Experts have selected manually five landmarks (micro calcifications, macro calcifications ...) present on both images. Distance before registration is computed by superimposing, in an absolute and global way, the two images of each couple, independently of their contents. In order to evaluate our results (see Table 1 and Fig.7), we compare them with those obtained with a global translation matching the nipples in the two images, as practiced, in a mental way, by radiologists.

The results show a diminution of the mean distance between the selected landmarks. Our method gives better results than a rigid registration either for mean distance between landmarks or standard deviation. Maximum error is also reduced (Fig. 7).

\begin{tabular}{|l|l|l|l|}
\hline \multicolumn{1}{|c|}{ Distance } & \multicolumn{1}{c|}{$\begin{array}{c}\text { Before } \\
\text { registration }\end{array}$} & $\begin{array}{l}\text { Translation } \\
\text { registration }\end{array}$ & $\begin{array}{c}\text { Our } \\
\text { method }\end{array}$ \\
\hline Mean & 1.02 & 0.68 & 0.62 \\
\hline $\begin{array}{l}\text { Standard } \\
\text { Deviation }\end{array}$ & 0.58 & 0.40 & 0.33 \\
\hline \hline $\begin{array}{l}\text { Mean execution } \\
\text { time (sec) }\end{array}$ & - & 3.05 & 3.95 \\
\hline
\end{tabular}

Table 1 - Comparative results: mean distance $(\mathrm{cm})$ and execution time (sec) for a couple of temporal mammograms 
The processing time of our method is higher than with translation registration but is still reasonable, it is far lower than the processing time of complex non-rigid methods, which is about 4 minutes in the most favorable case with parallel implementation [12].

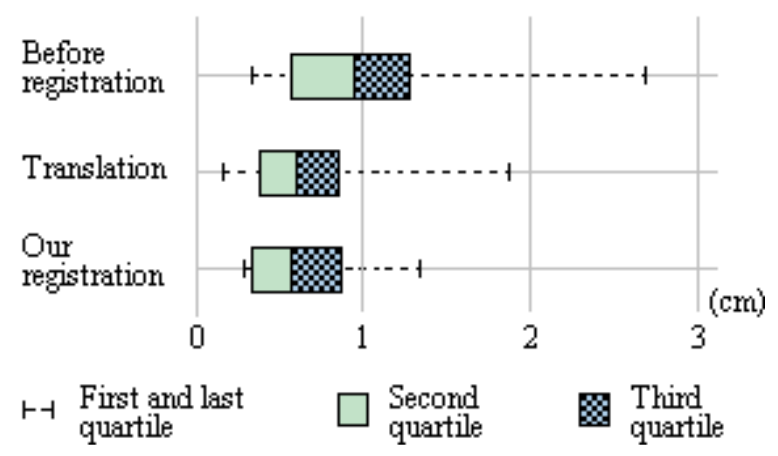

Figure 7 - Distance between couple of selected landmarks grouped in quartiles

\section{CONCLUSION}

We propose a fully automated method to register mammograms. This method is a coarse-to-fine approach. It is a globally rigid method based on robust anatomical features that minimizes differences caused by positioning and compression as much as possible. Some deformation based on linear scalings can then be applied locally, as some semantical meaning has been associated with each region at the previous step. This method preserves in mammograms differences really caused by breast changes and new disease appearance or progress of existing disease.

Future works will be devoted to define more precisely the non-rigid transforms. Indeed, the different lines are not independent and we will involve some texture elements to have an even more realistic vision.

\section{ACKNOWLEDGMENTS}

We would you like to thank the ANRT, the French National Agency of Research and Technology for its financial support. We also want to thank Dr A. Bremont for his valuable suggestions and inspiring conversations.

\section{REFERENCES}

[1] J. Suri, R. Rangavyyan, "Recent Advances in Breast Imaging, Mammography, and CAD of Breast Cancer", SPIE Press Book, 2006.

[2] L. Brown, "A survey of image registration techniques", ACM, Computing Surveys, 24(4), pp. 325-376, December 1992.

[3] M. Wirth, "A non-rigid approach to medical image registration: Matching image of the breast", Ph. D thesis, RMIT University Melbourne, 1999.

[4] S. Kwok, R. Chandrasekhar, Y. Attikiouzel, M. Rickard, "Automatic pectoral muscle segmentation on mediolateral oblique view mammograms", IEEE Trans. on Medical Imaging, 23(9), pp. 11291140, 2004.

[5] H. Mirzaalian, M. Ahmadzadeh, S. Sadri, "Pectoral muscle segmentation on digital mammograms by nonlinear diffusion filtering", IEEE P. RIM Conf. on Communications, Computers, and Signal Processing, pp. 581-584, 2007.

[6] M. Mustra, J. Bozek, M. Grgic, "Breast border extraction and pectoral muscle detection using wavelet decomposition", IEEE Eurocon, pp. 1426-1433, 2009.

[7] R. Woods, S. Cherry, J. Mazziota, "Rapid Automated Algorithm for Aligning and Reslicing PET Images", Journal of Computer Assisted Tomography, 16(4), pp. 620-633, July 1992.

[8] Y. Chiou, CS. Lin, CY. Lin, "Hybrid Registration of Corresponding Mammogram Images for Automatic Detection of Breast Cancer", Biomedical Engineering Applications Basis Com., 19(6), pp. 359374, 2007.

[9] V. Rajagopal, N. Nash, R. Highnam, P. Nielsen, "The breast biomechanics reference state for multi-modal image analysis", 5116 LNCS, pp. 385-392, 2008.

[10] A. Lee, V. Rajagopal, P. Bier, P. Nielsen, M. Nash, "Correlation of breast image alignment using bio-mechanical modeling", Progress in Biomedical Optics and Imaging - SPIE, Vol. 7262, $\mathrm{n}^{\circ}$ 726219, 2009.

[11] N. Betrouni, "Multimodality medical images registration : From conception to validation", IRBM 30 (2), pp. 60-71, April 2009.

[12] R. Stefanescu, X. Pennec, N. Ayache, "A Grid Service for the Interactive use of a Parallel Non-Rigid Registration Algorithm of Medical Images", Methods of Informations in Medicine, 44(2), 2005. 